\title{
Time-resolved Q-factor Measurement and its Application in Performance Analysis of 42.6 Gbit/s Packets Generated by SGDBR Lasers
}

\author{
Arvind K. Mishra, Andrew D. Ellis and Liam P. Barry
}

\begin{abstract}
We demonstrate a novel time-resolved Q-factor measurement technique and demonstrate its application in the analysis of optical packet switching systems with high information spectral density. For the first time, we report the time-resolved Q-factor measurement of $42.6 \mathrm{Gbit} / \mathrm{s}$ AM-PSK and DQPSK modulated packets, which were generated by a SGDBR laser under wavelength switching. The time dependent degradation of Q-factor performance during the switching transient was analyzed and was found to be correlated with different laser switching characteristics in each case.
\end{abstract}

Index Terms- SGDBR Laser, Wavelength-Switching characteristics, Optical Packet Switching, DQPSK

\section{INTRODUCTION}

$\mathrm{F}$ AST wavelength switching sampled-grating distributedBragg-reflector (SGDBR) lasers are expected to play a significant role in dynamic wavelength allocation and packet routing in optical packet switched or optical burst switched networks[1-4]. To enable efficient utilization of the available channel capacity in such networks, it is necessary to enhance the spectral efficiency of individual data channels, while minimising the necessary temporal guard-time. Using fast tuneable SGDBR lasers, a $2.5 \mathrm{Tbit} / \mathrm{s}$ capacity switch fabric was demonstrated at a data rate of $40 \mathrm{Gbit} / \mathrm{s}$ using NRZ modulation with information spectral density (ISD) of 0.4 $\mathrm{bit} / \mathrm{s} / \mathrm{Hz}$ [5]. Enhancement of the ISD to $0.8 \mathrm{bit} / \mathrm{s} / \mathrm{Hz}$ was achieved using duobinary modulation [6, 7]; however, the required guard-time to avoid performance degradation induced by laser switching characteristics [8, 9] was not reported, and measurements were restricted to $50 \%$ of the packet length. Whilst errors induced by rapid transient effects in wavelength switched networks may be avoided by

Manuscript received October 12, 2009. This material is based upon work supported by the Science Foundation Ireland under Grants 06/IN/I969 and 03/IN3/I560.

Arvind K. Mishra and Andrew D. Ellis are with the Photonic Systems Group, Tyndall National Institute and Department of Physics, University College Cork, Lee Maltings, Prospect Row, Cork, Ireland (e-mail: akmishra1@gmail.com, andrew.ellis@tyndall.ie).

Liam. P. Barry is with the Research Institute for Networks and Communications Engineering, School of Electronic Engineering, Dublin City University, Dublin 9, Ireland (e-mail: liam.barry@dcu.ie). increasing laser blanking times [10] this reduces the overall channel capacity. Measurements of the temporal location of error bursts within a packet may be correlated to dynamic characteristics of the laser, such as spurious emission or the instantaneous change in frequency and line-width, and this may allow identification and optimisation of laser characteristics which will maximise data throughput. Conventionally, such information is inferred from overall packet error-rate measurements $[10,11]$ or using commercial error detector gating facilities [12, 13]. However, such techniques offer restricted temporal resolution and require extended measurement times.

In this paper, we report a novel method to estimate the bit error rate (BER) and the time-resolved quality factor (Qfactor) of the modulated signal throughout the duration of a packet. We apply this method to $42.6 \mathrm{Gbit} / \mathrm{s}$ Amplitude Modulated Phase Shift Keying (AM-PSK) [14] and Differential Quaternary Phase Shift Keying (DQPSK) [15] formatted packets generated by SGDBR lasers and identify degradations due to the dynamics of the wavelength and linewidth respectively. The proposed scheme is independent of packet/burst length and in principle allows the location of error bursts.

The paper is organized as follows. Section II provides a detailed description of the time-resolved BER measurement setup. Results of Q-factor measurements of 42.6 Gbit/s AMPSK packets are provided as an example. In section III, a simple modification to the scheme to enable detection of phase modulated packets is presented, using DQPSK packets as an example. In section IV, we illustrate the identification of the dominant source of errors for both modulation formats. Finally in Section V we present our conclusions.

\section{TIME RESOLVED BER MEASUREMENT}

The approach for our time-resolved BER measurement technique is illustrated in figure 1 . The BER measurement section is formed from an error pulse detector and an error counter (signal integrator).

Within the error detection section, a signal under test is temporally aligned using optical delay lines with a reference signal carrying the same logical information. A clock signal is 
extracted from the reference data and used to time independent decision gates for the reference and test data. The outputs of the decision gates are compared in an exclusive OR (XOR) gate, which generates a binary output pulse whenever the two patterns are different. Each error pulse is considered to represent a single error in the test pattern. In its simplest form, the error counter would comprise a low pass filter with an impulse response $T_{D}$ followed by an analogue to digital converter. In this case, the recorded output would be the integral of the energy associated with each error pulse, and so the recorded voltage would be directly proportional to the number of errors received within a period $T_{D}$. By monitoring this voltage as a function of time, the BER of the incoming data burst may be estimated. In this experiment, three additional features were added. Firstly, since the XOR gate employed was un-timed, pattern dependent differences between the rising and falling edges of the signal and reference channels resulted in small spurious pulses at the XOR output. These pulses would also be integrated by the low pass filter, restricting the dynamic range of the BER estimation. To mitigate this effect, an additional high speed logic gate (OR) was employed to threshold the error pulses. This gate was followed by a further $1 \mathrm{GHz}$ analogue switch controlled by a gating signal following the packet envelope to only pass error pulses when the packet envelope is high to eliminate apparent errors between packets which would otherwise be counted. Finally, to relax the constraints placed on the ADC, the error pulses were low pass filtered using a logarithmic amplifier with a $100 \mathrm{MHz}$ bandwidth, such that the output signal was proportional to the logarithm of the number of errors detected in a 10ns period. Instead of an ADC, a low cost sampling oscilloscope with an 8-bit resolution enabled the estimated BER evolution to be monitored in real time for a given decision threshold of the switching arm D-type flip-flop (DFF). The resultant traces were transferred to a computer for processing using Labview 7.1.Note that the use of the AWG in this paper is to allow the generation of a time invariant reference signal carrying the same logical data. The measurement approach remains valid for other front end configurations, including the use of a power splitter for circumstances where error free operation was possible with a fixed decision threshold, or a parallel fibre path with appropriate delay adjustment.

To illustrate the operation of the system, we performed time resolved BER measurements of AM-PSK packets. The packet switched transmitter consisted of a wavelength switching tuneable SGDBR laser (TLS) module [16] followed by a Mach-Zehnder modulator (MZM). The TLS can switch between $80 \mathrm{C}$-band channels on the $50 \mathrm{GHz}$ ITU grid and was used to generate a continuous stream of optical data packets of variable duration by switching wavelength between $1553.45 \mathrm{~nm}$ and $1545.04 \mathrm{~nm}$. The MZM was biased at the minimum transmission and driven by a duobinary signal, which was derived from a 42.6Gbit/s NRZ signal of $2^{7}-1$ pseudorandom binary sequence (PRBS) length followed by duobinary amplifier $(15 \mathrm{GHz}$ bandwidth), producing a spectrally efficient 42.6 Gbit/s AM-PSK formatted signal. The pattern length was limited by the quality of our drive electronics. As a self referenced system, the performance of the $\mathrm{Q}$ factor measurement system itself should be independent of the transmitted pattern, and if appropriately triggered should even be able to temporarily locate dominant error patterns. A reference bit pattern was generated at a fixed wavelength of $1549.42 \mathrm{~nm}$ by launching the output of a CW laser (ECL) through the same modulator. At the receiver, the signal was optically pre-amplified before the channels were demultiplexed using a 50/100 $\mathrm{GHz}$ interleaver and a 100 GHz-spaced Gaussian-shaped arrayed waveguide grating (AWG). The continuous reference signal and packet data pattern were directed to the independent receivers and decision circuits with appropriately adjusted optical delays.

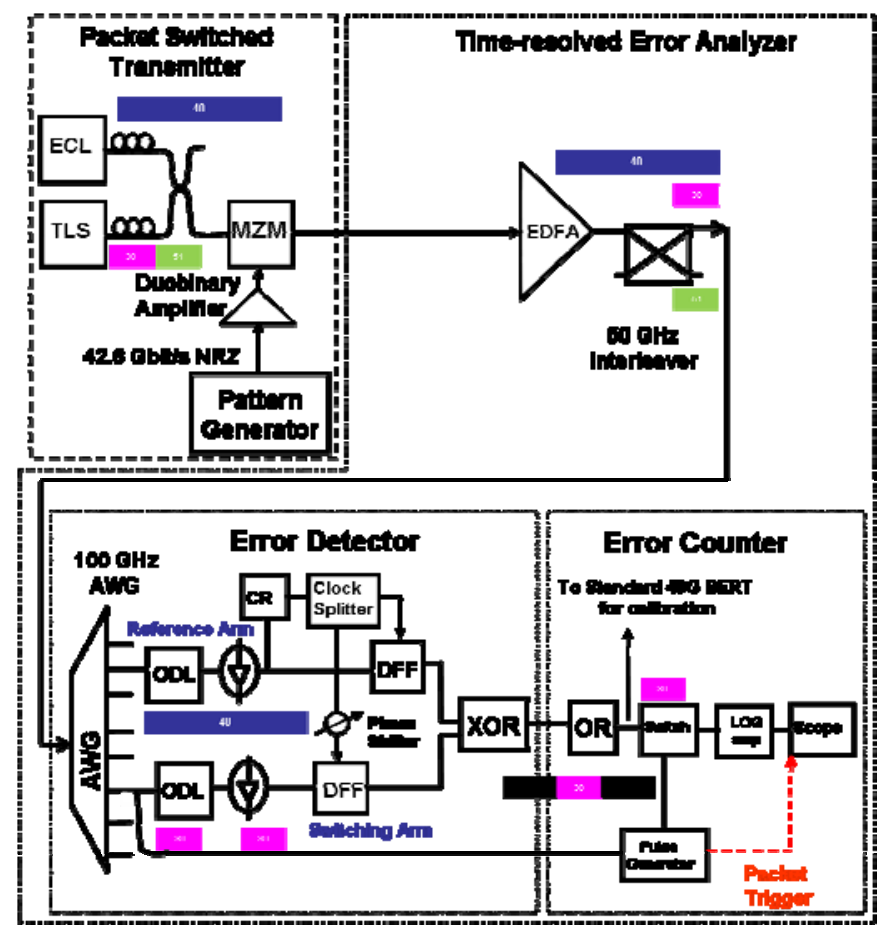

Figure 1: Experimental setup of $42.6 \mathrm{Gbit} / \mathrm{s}$ optical packet-switched transmitter and bit error analyzer; TLS: tuneable laser source, ECL: external cavity laser, ODL: optical delay line, CR: clock recovery unit, DFF: D-type flip-flop decision gate.

The eye diagram of the continuous signal and packet switching data are shown in figure 2(a) and 2(b), respectively, measured using a sampling oscilloscope without gating. In the reference arm, the decision threshold was fixed at the optimum generating the original data pattern without errors. The optical packet data is detected in the switching signal arm with an adjustable decision threshold to measure 3D data set of BER versus threshold during whole packet durations as described above, compared to the reference pattern using an XOR gate. 

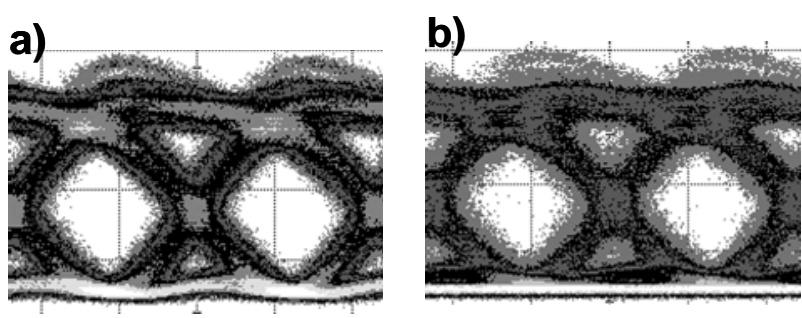

Figure 2: The eye diagram of ch\#30 modulated with $42.6 \mathrm{Gbit} / \mathrm{s}$ AM-PSK signal (a) for continuous data, and (b) $10 \mu$ s packet of ch\#30 switching from ch\#51 with laser output blanked and locker engaged.

Figure 3 illustrates typical outputs of the error detection block (XOR gate) for four different decision thresholds, and shows the error pulses (top) and histograms of their distributions for $10 \mu \mathrm{s}$ packets at $1553.45 \mathrm{~nm}$ following a switching transition from $1545.04 \mathrm{~nm}$. In addition to showing no recorded errors at the optimum threshold voltage of -70 $\mathrm{mV}$ (fig 3(c)), this figure illustrates the temporal distribution of errors throughout the packet for different threshold voltages. In this example, errors in the 1 level (omissions) dominate at the beginning of the packet (fig 3(b)) and errors in the 0 level (insertions) at the end (fig 3(d)).

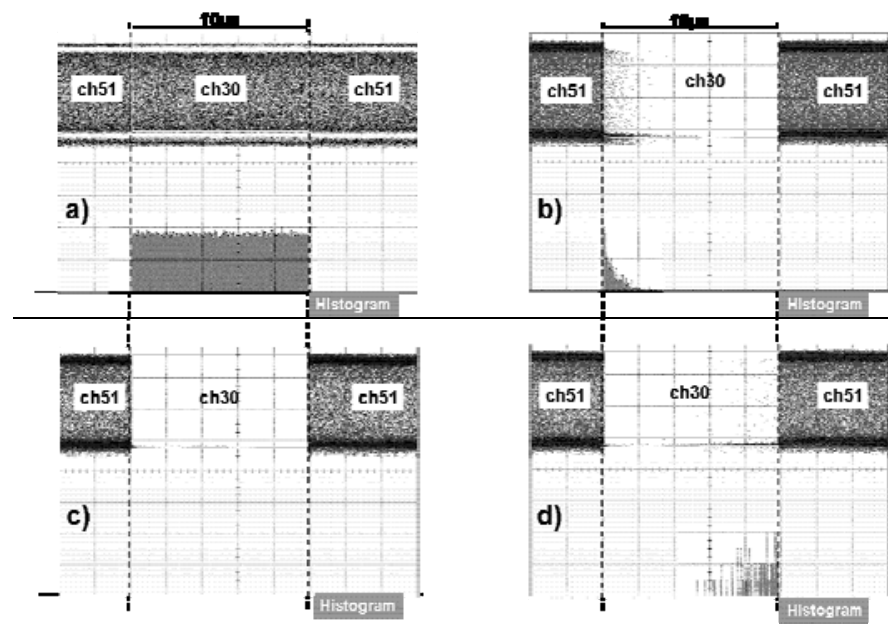

Figure 3: Output of XOR gate, ' 1 ' level representing error bits and corresponding error distribution using scope in histogram mode of $42.6 \mathrm{Gbit} / \mathrm{s}$ AM-PSK modulated $10 \mu$ s 1553.45 nm packet of PRBS length of $2^{7}-1$ at decision thresholds of (a) $+40 \mathrm{mV}$ (“completely errored”); (b) $-27 \mathrm{mV}$ (“omission errors”); (c) $-70 \mathrm{mV}$ ("no errors observed”); (d) $-110 \mathrm{mV}$ ("insertion errors").

In order to calibrate the time resolved BER measurement, a CW signal was applied to the switching arm of the error detector. With no signal applied to the reference arm, the detected signal pattern was reproduced at the OR gate output. This pattern was directed to a commercial BER detector via an error-free optical link enabling the actual BER to be recorded. Then, with a signal applied to the reference arm, the output voltage from the logarithmic amplifier was measured. The process was repeated approximately five times by varying the decision threshold of the signal DFF to obtain the desired range of BERs. A typical calibration curve is shown in figure 4(a), and may be used to correct deviations from the expected logarithmic ratio of amplitude output to BER values arising either from the noise floor of components in the error counter section at low BER $\left(<10^{-9}\right)$, or from multiple error pulses occurring within the duration of a single output pulse from the final logic gate (error collisions) at high BER $\left(>10^{-4}\right)$. Note that to increase the accuracy of the calibration curve, the error voltage was integrated over a number of measurements. Using a curve fit of the calibration data, the voltage variation at the output of the error counter section may be converted to an estimated BER variation. A selection of such time-resolved bit error rate measurements at different threshold voltages are shown in figure 4(b).
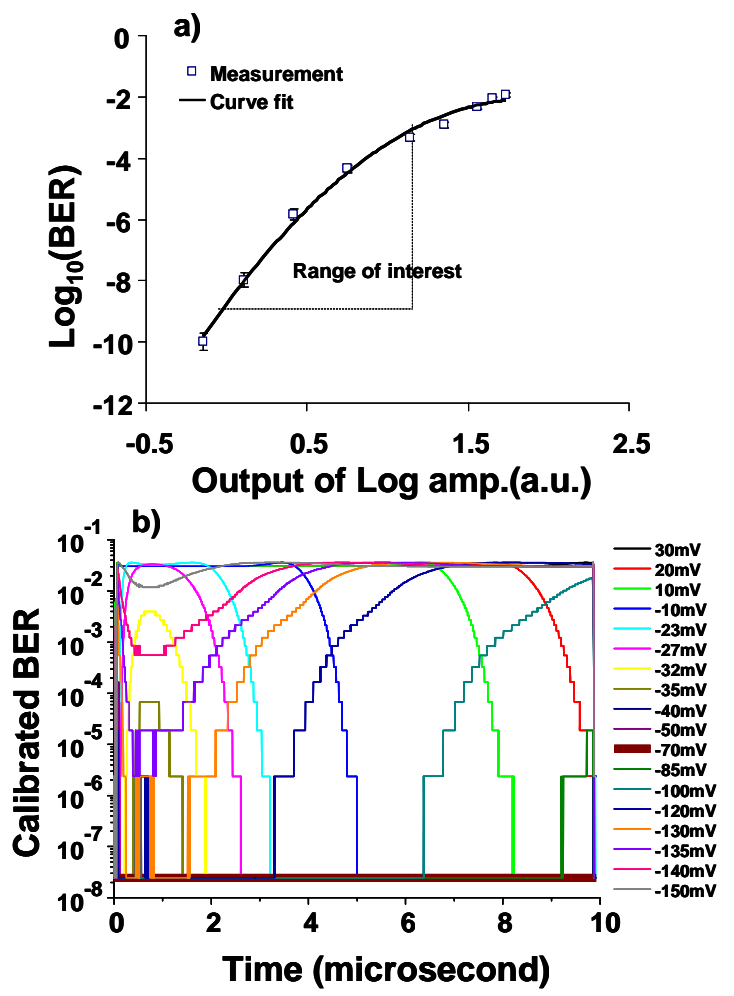

Figure 4(a): Measured and curve fitted BER versus error voltage; (b) Calibrated instantaneous BER of $10 \mu \mathrm{s}$ ch\#30 packet at various decision thresholds of switching arms DFF.

Combining time traces of BER measurements taken at different decision thresholds, the BER versus threshold of the packets at any given moment after a switching event can be obtained by time slicing this 3D data set parallel to the threshold axis. In figure 5, a typical set of BER versus threshold curves are shown. The Q factor was calculated as the electrical signal to noise ratio from the mean and standard deviation of the 'zero' and 'one' levels using the conventional formula using [17-20]. Such results enable the Q-factor of the signal to be measured at any time within the packet by fitting the BER versus threshold curves in the usual manner. 


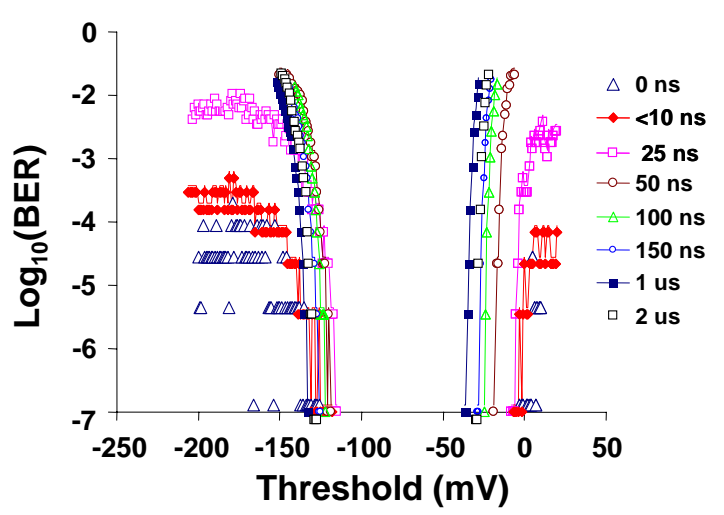

Figure 5: BER versus threshold curve for $10 \mu$ s packet of ch\#30 at different time interval just after blanking (blanking duration of $64 \mathrm{~ns}$ is not included in time).

The time resolution of the measurement technique was verified by amplitude modulating a continuous signal with an SOA driven with a square wave signal, and measuring the time delay between the SOA signal and the recorded Q factor variation. This indicated that the measurement system had an impulse response of 10 ns, as expected from the analogue bandwidth of the system.

Figure 6 shows time-resolved Q-factor measurements for 42.6 Gbit/s AM-PSK modulated $10 \mu \mathrm{s}$ optical packets generated as described above. The figure shows the $\mathrm{Q}$ factor evolution with and without blanking of the laser output using an integrated semiconductor optical amplifier (SOA), previously demonstrated to suppress spurious modes during establishment of the correct operating currents. It also shows the impact of the laser wavelength locking circuit.

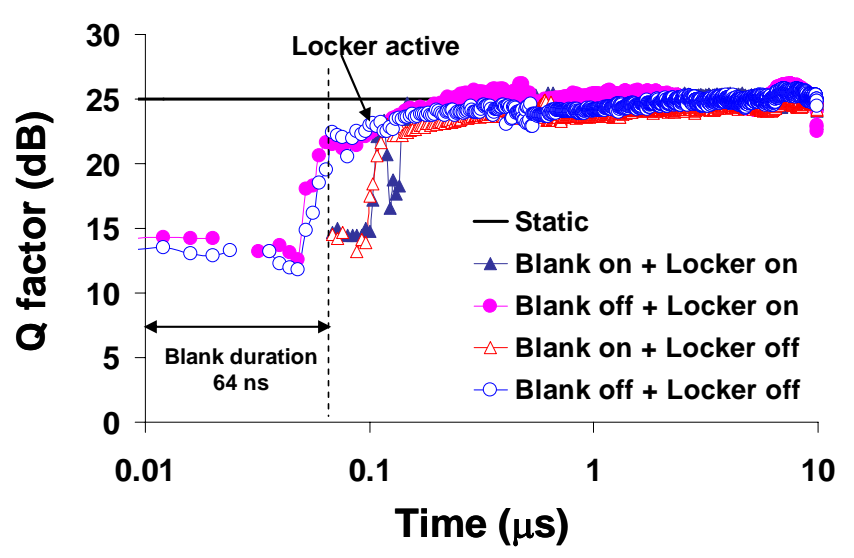

Figure 6: Time-resolved Q-factor measurements of 42.6 Gbit/s AM-PSK modulated $10 \mu$ s packet at $1553.45 \mathrm{~nm}$ generated by SGDBR laser module switching from 1545.04nm with, SOA blanking enabled (triangles) or disabled (circles) and with wavelength locker enabled (filled symbols) or disabled (open symbols).

The severe degradation in $\mathrm{Q}$ factor, observed at lower bit rates [13] induced by spurious modes during the initial switching transient is clearly observed during the first 60ns after switching. With the blanking enabled, no $Q$ factor measurements are possible until emission occurs, after which time the Q factor gradually reaches its steady state value as the laser settles down following the switching transient. In this case however, a subsequent error burst is evident corresponding to the delayed activation of the wavelength locker. In all cases, a degradation in Q factor at the end of the packet is observed, which is attributed to tuning current noises arising from signaling within the module in preparation for the next wavelength switching event.

\section{DQPSK PACKETS}

Unlike a conventional bit error rate test set, the approach adopted in this paper does not require the independent generation of a local copy of the received data pattern, since this is directly generated in the reference arm from a continuous copy of the transmitted data. This is particularly advantageous for higher order modulation formats, where this self referenced behaviour removes the need for programming of the error detector with the expected data sequence. To access higher order modulation formats, it is necessary to precede the Q factor analyser with an optical demodulator appropriate to the signal under test. In order to demonstrate this feature, we also performed measurements of DQPSK packets, generated from a second SGDBR laser by adding a one bit delay interferometer. The experimental setup is illustrated in figure 7. The packet transmitter consisted of a tuneable SGDBR laser followed by a DQPSK modulator. The TLS was used to generate optical data packets of duration 10 $\mu$ s by switching wavelength between $1553.4 \mathrm{~nm}$ and 1545.0 nm. A $2^{7}-1$ PRBS data signal at $21.3 \mathrm{Gbit} / \mathrm{s}$ was generated by multiplexing two $10.65 \mathrm{Gbit} / \mathrm{s}$ signal outputs of the pattern generator each fed to two inputs of a 4:1 40G multiplexer. The non-inverted and inverted output $21.3 \mathrm{Gbit} / \mathrm{s}$ NRZ signals were used as I and Q signals to drive the DQPSK modulators, where the I signal was delayed using one meter RF ( 70 symbol periods) cable to further de-correlate the patterns. A reference bit pattern was generated at a fixed wavelength of $1549.4 \mathrm{~nm}$ by launching the output of a CW laser through the same modulator.

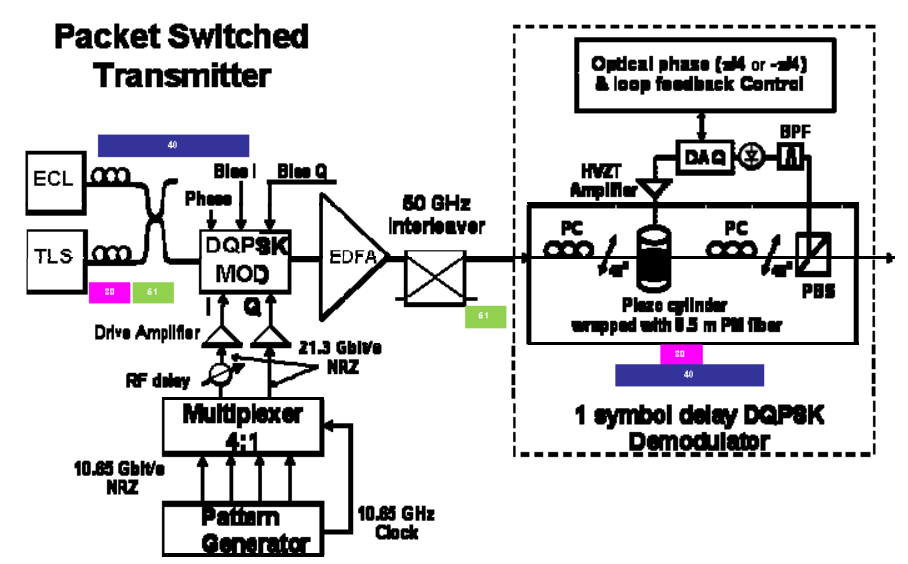

Figure 7: Experimental setup of $42.6 \mathrm{Gbit} / \mathrm{s}$ DQPSK modulated optical packet-switched transmitter (a); and time-resolved receiver (b). TLS: tuneable laser source, ECL: external cavity laser, ODL: optical delay line, CR: clock recovery unit. 
The DQPSK signals were demodulated using a one symbol delay-and-add interferometer constructed from polarization maintaining fiber. The PM fiber was wrapped on a piezo cylinder to enable phase bias of the interferometer and selection of the I or Q quadrature for the single ended detection using the error detector described above. An appropriate wavelength for the reference signal was selected to ensure that demodulation of signals directed to the reference and signal arms represented the same quadrature. (a)

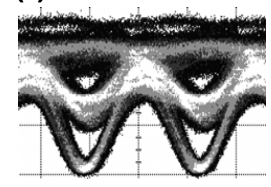

(b)

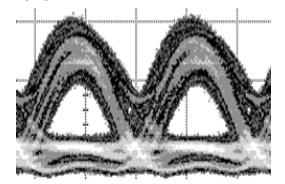

(c)

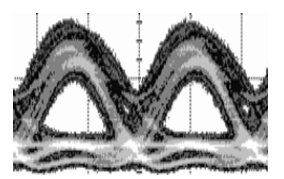

Figure 8: Eye diagrams of $42.6 \mathrm{Gbit} / \mathrm{s}$ DQPSK signals (a) transmitted; and demodulated-I channel of $42.6 \mathrm{Gbit} / \mathrm{s}$ DQPSK signal (b) switching arm, (c) reference arm with the continuous data of PRBS length of $2^{7}-1$ (Time axis 20 ps/Div).

The eye diagrams for the transmitted $42.6 \mathrm{Gbit} / \mathrm{s}$ DQPSK signals are shown in figure 8(a) and eye diagrams of demodulated switching arm and reference arm signal with continuous data are shown in figure 8(b) and 8(c), respectively. A typical calibration curve for DQPSK data is shown in figure 9, where the expected exponential relationship (note logarithmic scale on $\mathrm{x}$ and $\mathrm{y}$-axis) is observed over 6 orders of magnitude of BER.

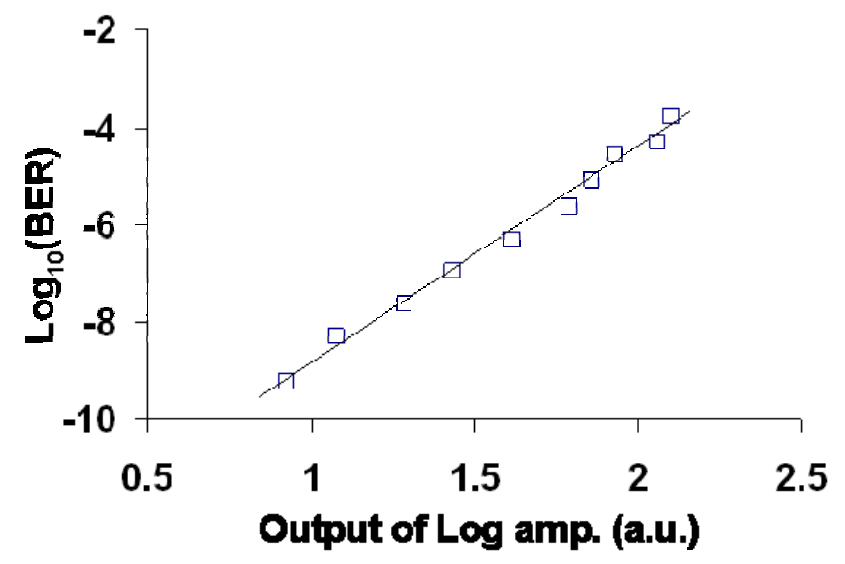

Figure 9: Measured and fitted BER versus error voltage.

Typical measured time-resolved Q-factors for SGDBR DQPSK packets of $10 \mu$ s duration are shown in figure 10 . Without SOA blanking the BER was initially very high and Q-factor estimation was only possible after a delay of 60ns, during the laser settling time (400ns) oscillatory error bursts were observed. Similar Q-factor results were found with SOA blanking enabled for a period of $64 \mathrm{~ns}$, although the action of the locker perturbed the initial transient behavior to some degree

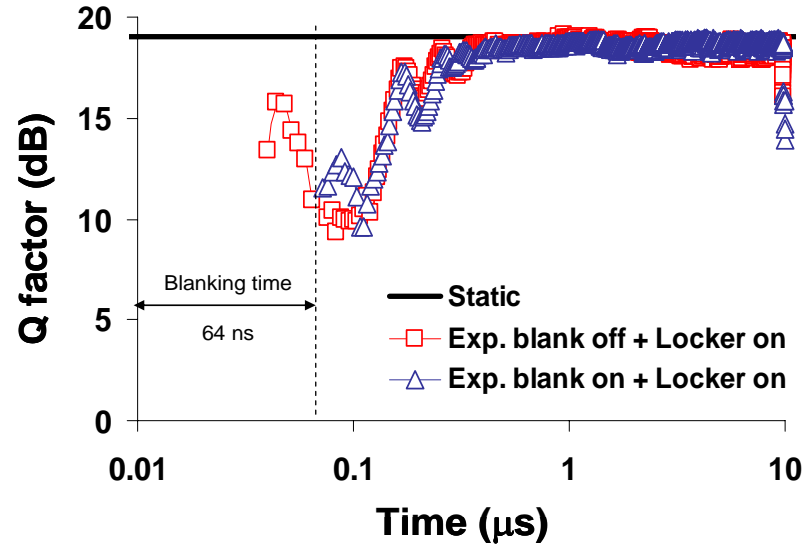

Figure 10: Time-resolved Q-factor measurement of $10 \mu \mathrm{s}$ ch\#30 DQPSK modulated packet of PRBS $2^{7}-1$ for SGDBR laser module switching from ch\#51.

The Q-factor dynamics of packets were further investigated for other channels covering a wide range separation between switching channels from the target channel. The time-resolved Q-factor measurement results follow the similar dynamics for all switching combinations. However, the initial Q-factor magnitude was different with a variation of 5-10 dB depending on the switching combination, which would directly depend on the amount of current injection required for the relative separation between target and source channel.

\section{PERFORMANCE ANALYSIS OF PACKETS}

To illustrate the usefulness of time resolved error rate measurements, we demonstrate the correlation of the observed error bursts with physical dynamics of the laser emission. We consider three basic features of the laser output: the instantaneous optical frequency which dictates the total power transmitted through the demultiplexing filters; the instantaneous linewidth which directly induces bit errors for phase modulated systems; and phase to amplitude noise conversion induced by the action of the filters on the dynamic laser linewidth.

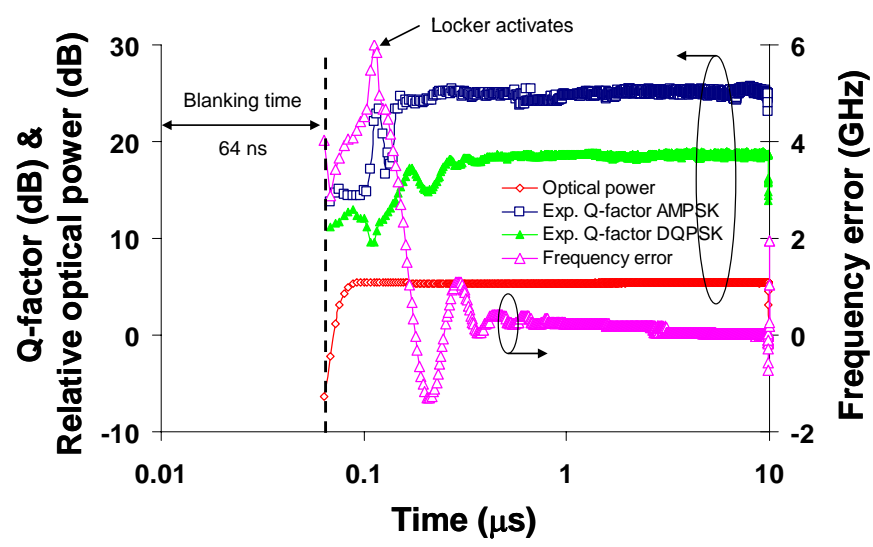

Figure 11: Measured time-resolved frequency error and the relative optical power along with time resolved Q-factor measurement results of AM-PSK and DQPSK modulated packets of duration of $10 \mu$ s of ch\#30 with default blanking duration of 64 ns and locker active. 
This article has been accepted for publication in a future issue of this journal, but has not been fully edited. Content may change prior to final publication. $>$ REPLACE THIS LINE WITH YOUR PAPER IDENTIFICATION NUMBER (DOUBLE-CLICK HERE TO EDIT) <

Figure 11 illustrates the time-resolved frequency error and modulated power transmitted through the demultiplexing filters, along with the time resolved $\mathrm{Q}$ factor for both modulation formats. Clearly in both cases the transmitted power has stabilised more rapidly than the Q factor, strongly indicating that penalties from attenuation of the modulation sidebands do not dominate the observed BER variations. For the linewidth dependant errors, we follow standard analysis for phase shift keyed signals [26] and phase noise to intensity noise conversion [22, 23] based on temporarily invariant linewidths.

Phase-to-intensity-noise conversion while passing through highly dispersive material is well known, and in our case arises from the trapezoidal shaped profile interleaver $(25 \mathrm{GHz}$ pass-band and $80 \mathrm{GHz}$ stop-band attenuation of $30 \mathrm{~dB}$ ). In this case, an additional noise variance $\sigma_{\Delta v}^{2}$ is added to the marks:

$$
\sigma_{\Delta v}^{2}(t)=\frac{32}{3} R \pi^{3} F^{2} B^{3} P_{i n}^{2} \cdot \Delta v(t)
$$

where, $R$ is responsivity of the detector, $B$ is bit rate of signal, $P_{i n}$ is optical power input through the dispersive material, $\Delta v$ is the time dependent FWHM linewidth of the laser, and $F=\frac{D \cdot \lambda^{2}}{2 \pi \cdot c}$ is the dispersion parameter $\left(\mathrm{s}^{2}\right)$ of the interleaver, where $\mathrm{D}$ is the dispersion $(\mathrm{s} / \mathrm{m})$ of the interleaver. The receiver bandwidth is assumed to be the same as the data rate. The dispersion parameter of the interleaver was measured using the phase shift method [24] and is shown in figure 12 along with a trapezoidal shaped filter module that was used for numerical simulation of this system using VPI transmission maker 7.5.

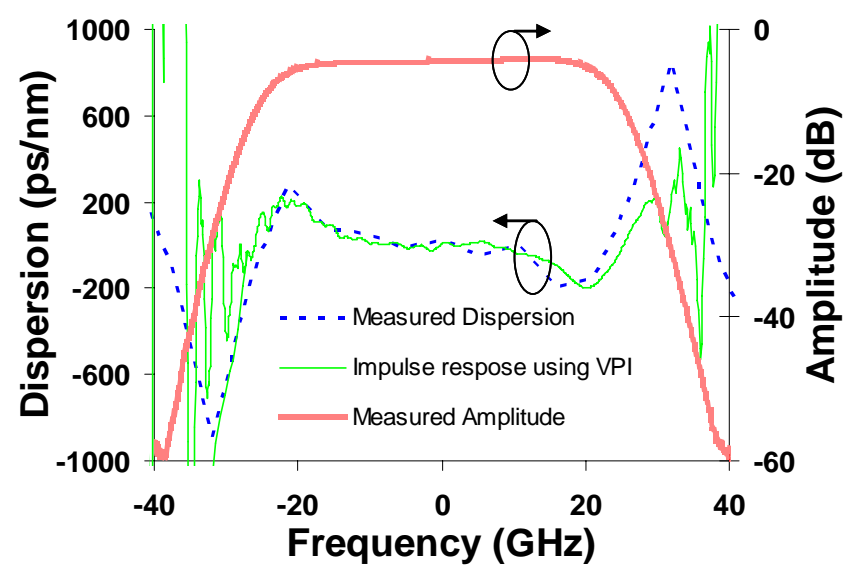

Figure 12: Measured dispersion parameter and amplitude response of $50 \mathrm{GHz}$ interleaver and dispersion parameter used for numerical simulation.

The impact of excess laser linewidth on Q-factor of the 42.6 Gbit/s AM-PSK signal passing through a $50 \mathrm{GHz}$ interleaver (section II) was theoretically analyzed including equation (1) with the parameters listed in table-I (figure 13) using a value of $0.027 \mathrm{~s} / \mathrm{m}$ for the filter dispersion, taken from the centre of the passband. The laser FWHM linewidth was measured using heterodyne detection and an estimate was made of the apparent linewidth that would be induced by centre frequency drift (by differentiating the frequency error measurements and integrating over the measurement interval) [25].

\begin{tabular}{|c|c|c|}
\hline Parameters & ANPEK & DQPEK \\
\hline Symbol rate (B) & $42.6 \mathrm{~Gb} / \mathrm{s}$ & $21.3 \mathrm{~Gb} / \mathrm{s}$ \\
\hline IIme delay (I) & - & $4 / \mathrm{FS}$ \\
\hline $\begin{array}{l}\text { Dispersion (D) } \\
\text { (FDeod valuo) }\end{array}$ & $0.027 \mathrm{sm}$ & - \\
\hline OSNR input & $60 \mathrm{~dB}$ & $40 \mathrm{BB}$ \\
\hline EDFA yairı & $33 \mathrm{UB}$ & $38 \mathrm{CB}$ \\
\hline Noise Figure of EDFA & $50 \mathrm{~dB}$ & $5.0 \mathrm{cB}$ \\
\hline Phobodiade sensilivity & $-20 \mathrm{dBm}$ & $-20 \mathrm{dBm}$ \\
\hline Responsivity of phobodiode & $0.05 \mathrm{AW}$ & $0.8 \mathrm{AW}$ \\
\hline Oplical power input to EDFA & $-220 \mathrm{dBm}$ & $-26.5 \mathrm{dBm}$ \\
\hline
\end{tabular}

Figure 13: Table-I with a list of parameter used for theoretical calculation for AM-PSK and DQPSK signals.

The calculated Q factor (using measured FWHM linewidth and apparent linewidth) and measured Q-factors are presented in figure 14 for $10 \mu$ s packets with laser output blanked and locker engaged. Since linewidth measurement was not possible at a time scale lower than 80 ns due to limitation on the equipment used, therefore, the calculated Q-factor is presented after the 80 ns from the switching events excluding blanking duration of 64 ns. The calculated results with measured FWHM linewidth are found to be in good agreement with the measured data throughout the packet duration, and were not strongly associated with the dynamics of the apparent linewidth. The linewidth measurement step size was insufficient to resolve the fine structure observed in the $\mathrm{Q}$ factor evolution.

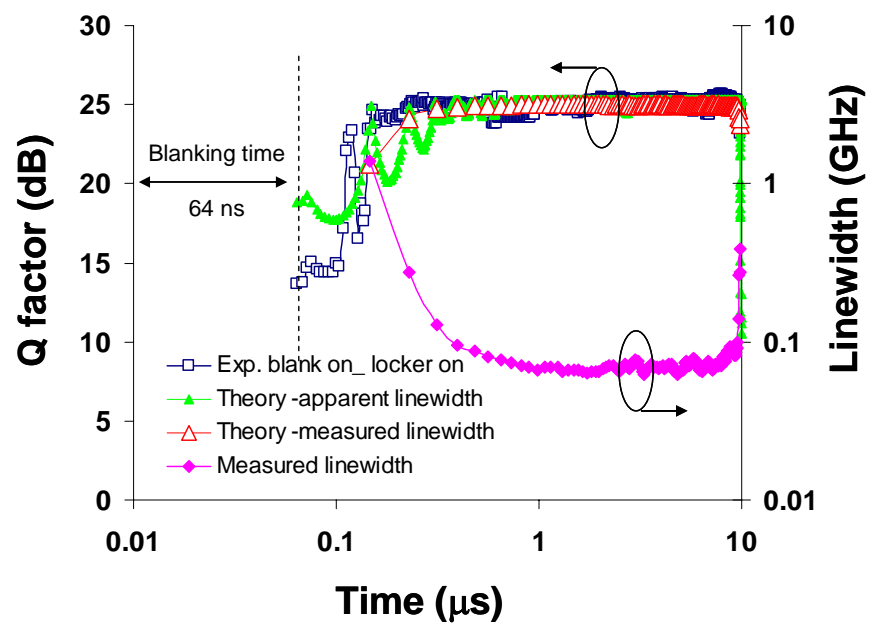

Figure 14: Plot of measured and calculated time-resolved Q-factor of 42.6 Gbit/s AM-PSK packets versus corresponding apparent linewidth and measured FWHM linewidth passing through $50 \mathrm{GHz}$ interleaver signal with blanking laser output and wavelength locker active.

Differential direct detection of a phase modulated signal system is susceptible to interferometric phase-to-intensitynoise conversion. In a wavelength switched system, the 
contribution of phase induced intensity noise at the output of the demodulator varies during, and immediately after switching. For a static system, the photocurrent variance as a result of interferometric intensity noise due to laser phase noise at the output of the DQPSK demodulator is given by [26]:.

$$
\sigma_{\Delta v-I}^{2}(t)=\frac{\left(R \cdot P_{\text {out }}\right)^{2} \cdot e^{-2 \cdot \Delta v \cdot T}}{16}\left\{\begin{array}{l}
\cos ^{2}\left(\omega_{0} T\right)[\cosh (2 \cdot \Delta v \cdot T)-1] \\
+\sin ^{2}\left(\omega_{0} T\right) \sinh (2 \cdot \Delta v \cdot T)
\end{array}\right\}
$$

where $R$ is the responsivity of the detector, $\omega_{0}$ is the peak optical frequency of the laser output of the interleaver, $T$ is the difference in time delay between the two arms of the demodulator, $P_{\text {out }}$ is the total output optical power of the EDFA, and the effects of time dependent linewidth and frequency error were combined in a single linewidth parameter as apparent linewidth $\Delta v$ of the laser and was obtained by differentiating the frequency error measurements and integrating over the measurement interval.

The DQPSK performance was analysed using the parameters listed in table-I (figure 13). The combined effects of instantaneous linewidth and frequency offset were combined in a single linewidth parameter [25], or measured FWHM linewidth used as inputs to equation (2). The measured time-resolved Q factor of DQPSK packets with laser blanking and wavelength locker active, are shown in figure 15. The temporal locations of the error bursts are found to be in good agreement with the theoretical results and the magnitudes are in close agreement after 120ns from the switching event. Our study confirms that the damped oscillatory feature of Q-factor dynamics of DQPSK packets were dominated by time dependent apparent linewidth as a combined effect of rate of change of frequency error in addition to degradation by the actual linewidth dynamics.

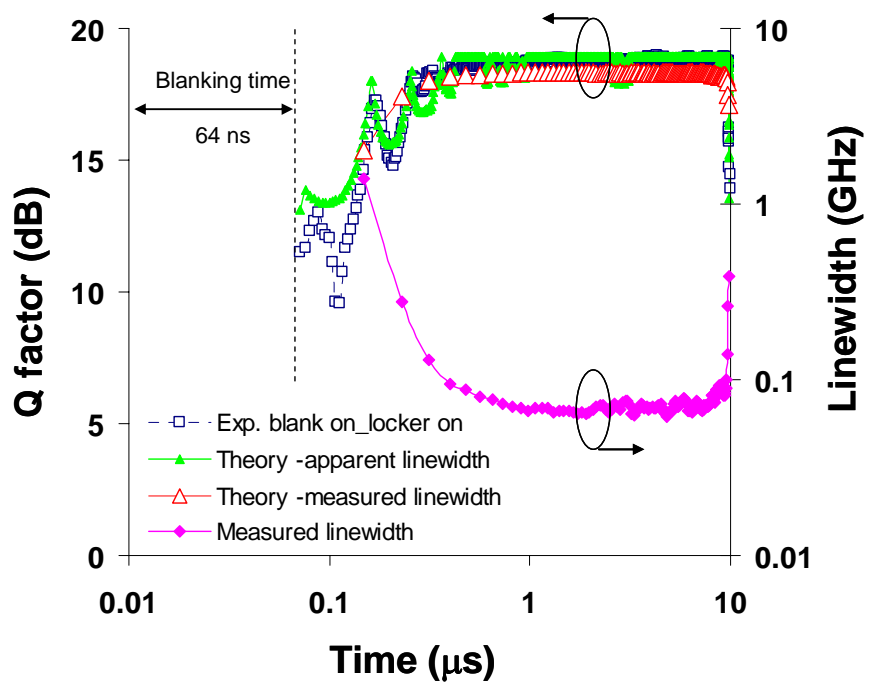

Figure 15: Experimental and calculated time-resolved Q-factor of $42.6 \mathrm{Gbit} / \mathrm{s}$ DQPSK packets with laser blanking and wavelength locker active versus corresponding apparent linewidth and measured FWHM linewidth.

\section{CONCLUSION}

We have demonstrated a novel technique for the timeresolved Q-factor measurement of packets compatible with measurements of burst-mode systems. This approach provides real time monitoring of the performance of burst mode systems, allowing for reductions in the development cycle of future wavelength switched systems. The time-resolved Qfactor was investigated for the first time for $42.6 \mathrm{Gbit} / \mathrm{s} \mathrm{AM-}$ PSK and DQPSK modulated optical packets generated by SGDBR lasers. We observed that the transient performance of both systems was dominated by the excessive phase noise of the laser following switching events. The use of advanced modulations formats and detection of the optical phase in wavelength switched systems would thus appear to require optimisation of the tuneable lasers to address the frequency drift and phase dynamics. Our results showed that these SGDBR lasers can be used for data transport in packet routed optical networks employing AM-PSK and DQPSK formats at the cost of temporal utilization of the channel bandwidth, where packet size is determined by the guard time (140-400 ns) needed with the desired Q-factor performance at the receiver. This is determined primarily by the laser tuning dynamics, but is influenced by the design of the tuning circuits. Reduction of the guard time would primarily require development of new tuneable laser sources with faster transition times and improved line-width [28].

\section{ACKNOWLEDGMENTS}

The authors would like to acknowledge Prof. David Cotter for useful discussion.

\section{REFERENCES}

[1] M.J. O'Mahony, D. Simeonidou, D.K. Hunter, A. Tzanakaki, “The application of optical packet switching in future communication networks", IEEE Communications Magazine, vol. 39, no. 3, pp. 128135, Mar. 2001.

[2] S. J.B. Yoo, "Optical packet and burst switching technologies for the future photonic Internet”, J. Lightwave Technol., vol. 24, no. 12, pp. 4468-4492, Dec.2006.

[3] D.J. Blumenthal, B.E. Olsson, G. Rossi, T.E. Dimmick, et al., "Alloptical label swapping networks and technologies”, J. Lightwave Technol., vol. 18, no. 12, pp. 2058-2075, Dec. 2000.

[4] K.V. Shrikhande, I.M. White, M.S. Rogge, F.-T. An, et al., "Performance demonstration of a fast-tunable transmitter and burstmode packet receiver for HORNET", in Proc. of. Optical Fiber Communication Conference (OFC’01), ThG2, Mar. 2001.

[5] J. Gripp, M. Duelk, J. Simsarian, P. Bernasconi, A. Bhardwaj,K. Sherman, K. Dreyer, M. Zirngibl, O. Laznicka, " $4 \mathrm{x} 4$ Demonstration of a $1.2 \mathrm{~Tb} / \mathrm{s}(32$ x $40 \mathrm{~Gb} / \mathrm{s})$ Optical Switch Fabric for Multi-Tb/s Packet Routers”, in Proc. ECOC 2002 , PD2.4, Sept. 2002.

[6] M. Duelk, J. Gripp, J. Simsarian, A. Bhardwaj, P. Bernasconi, M. Zirngibl, O. Laznicka, "Fast Packet Routing in a $2.5 \mathrm{~Tb} / \mathrm{s}$ Optical Switch Fabric with $40 \mathrm{~Gb} / \mathrm{s}$ Duobinary Signals at $0.8 \mathrm{~b} / \mathrm{s} / \mathrm{Hz}$ Spectral Efficiency”, in Proc. OFC 2003, PD8-1, Mar. 2003.

[7] J. Gripp, P. J. Winzer, G. Raybon, J. E. Simsarian, and C. R. Doerr, "107-Gb/s Serial Optical Packet Switching With 1-bit/s/Hz Spectral Efficiency for 100-GbE Backplanes”, IEEE Photon. Technol. Letters, vol. 19, no. 15, pp.1124-1126, Aug. 2007.

[8] H. Kim, and P.J. Winzer, "Robustness to Laser Frequency Offset in Direct-Detection DPSK and DQPSK Systems”, J. of Lightwave Technol., vol. 21, no. 9, pp. 1887-1891, Sep. 2003. 
[9] S. Savory and A. Hadjifotiou, "Laser Linewidth Requirements for Optical DQPSK Systems", IEEE Photon. Technol. Letters, vol. 16, no. 3, pp. 930-932, March 2004.

[10] E. Connolly, A. Kaszubowska and L.P. Barry, "Cross-Channel Interference Due to Wavelength Switching Events in Wavelength Packed Switched WDM Networks", in Proc. ECOC 2005, We1.4.6, Sept. 2005

[11] E. Connolly, F. Smyth, A.K. Mishra, A. Kaszubowska-Anandarajah, L.P. Barry, "Cross-Channel Interference Due to Wavelength Drift of Tunable Lasers in DWDM Networks”, IEEE Photon. Technol. Letters, vol. 19, no. 8, pp. 616-618, Apr. 2007.

[12] T.A. Lindsay, "Innovations in BER testers enable fast and accurate eye diagram, eye mask, Q-factor, and jitter measurements”, in Proc. OFC 2004, ThH4, Feb.2004

[13] F. Smyth, E. Connolly, A.K. Mishra, A.D. Ellis, D. Cotter, A. Kaszubowska, L.P. Barry, "Effects of Crosstalk in WDM Optical Label Switching Networks Due to Wavelength Switching of a Tunable Laser", IEEE Photonics Technology Letters, vol. 18, no. 20, pp.2177-2179, Oct. 2006.

[14] T. Ono, Y. Yano, K. Fukuchi, T. Ito, H. Yamazaki, et al., "Characteristics of Optical Duobinary Signals in Terabit/s Capacity, High-Spectral Efficiency WDM Systems”, J. of Lightwave Technol., vol. 16, no. 5, pp. 788-797, May 1998.

[15] R.A. Griffin and A.C. Carter, "Optical differential quadrature phase shift key (oDQPSK) for high-capacity optical transmission”, in Proc. of Optical Communication Conference (OFC’02), WX6, Mar. 2002.

[16] Intune AltoNet $1200 \quad$ FTL $\quad$ Tx $\quad$ Module, http://www.intunenetworks.eu/site/

[17] D. Marcuse, "Derivation of Analytical Expressions for the Bit-Error Probability in Lightwave Systems with Optical Amplifiers," J. Lightwave Technol., vol. 8, no. 12, pp. 1816-1823, 1990.

[18] D. Derickson, Fiber optic test and measurement, Prentice-Hall, Inc., 1998, p. 313.

[19] R. Hui, M. O'Sullivan, “Fiber Optic Measurement Techniques”, Elsevier Academic Press.

[20] G.P. Agrawal, "Fiber-Optic Communication Systems”, John Wiley \& Sons, NewYork, 1997.

[21] M.J. O'Mahony, I.D. Henning, "Semiconductor Laser Linewidth Broadening due to 1/f Carrier Noise”, Electronics Letters, vol. 19, no. 23, pp. 1000-1001, Nov. 1983.

[22] S. Yamamoto, N. Edagawa, H. Taga, Y. Yoshida, and H. Wakabayashi, "Analysis of laser phase noise to intensity noise conversion by chromatic dispersion in intensity modulation and direct detection optical-fiber transmission”, J. Lightwave Technol., vol. 8, no. 11, pp, 1716-1722, Nov. 1990.

[23] K. Petermann, J. Wang, "Large Signal Analysis of FM-AM Conversion in Dispersive Optical Fibres and its Application to PCM Systems", Electronics Letters, vol. 27, no. 25, pp. 2347-2348, Dec. 1991.

[24] S. Ryu, Y. Horiuchi, and K. Mochizuki, "Novel chromatic dispersion measurement method over continuous gigahertz tuning range”, J. Lightwave Technol., vol. 7, no.8, pp. 1177-1180, Aug. 1989.

[25] A.K. Mishra, A.D. Ellis, L.P. Barry, T. Farrell, "Time-Resolved Linewidth Measurements of a Wavelength Switched SG-DBR Laser for Optical Packet Switched Networks”, in Proc. OFC 2008, OTuC4, Feb. 2008.

[26] B. Moslehi, "Noise Power Spectra of Optical Two-Beam Interferometers Induced by the Laser Phase Noise”, J. Lightwave Technol, vol. LT-4, no. 11, pp. 1704-1710, NOV. 1986.

[27] David W. Smith, "Techniques for Multigigabit Coherent Optical Transmission”, J. Lightwave Technol., vol. LT-5, no. 10, pp. 1466-1478, Oct. 1987.

[28] R. Phelan, W-H. Guo, Q. Lu, D. Byrne, B. Roycroft, P. Lambkin, B. Corbett, F. Smyth, L. P. Barry, B. Kelly, J. O’Gorman, and J. F. Donegan, "A Novel Two-Section Tunable Discrete Mode Fabry-Pérot Laser Exhibiting Nanosecond Wavelength Switching”, J. Quantum Electron., vol. 44, no. 4, 331-337, April 2008.

Arvind K. Mishra was born in Varanasi, India in 1975. He received the M.Sc. degree in physics and the M.Tech. degree in optoelectronics and optical communication from the Indian Institute of Technology (IIT), New Delhi, in 1999 and 2001, respectively. He is presently working towards a Ph.D. degree in the area of applications of tuneable lasers in high capacity optical networks with the Photonic Systems Group, University College Cork, Ireland.
During 2001-2002, he was with Philips Research, Eindhoven, The Netherlands, as a research scientist in the ubiquitous communication system group, where he was involved in research on photonic components and network architectures for in-home networks. From November 2002-July 2005, he was working with Eindhoven University of Technology, Eindhoven, Netherlands, as a research assistant. His research was mainly focused on ultrafast nonlinear carrier dynamics of semiconductor optical amplifiers and its application to realize all-optical switching, wavelength conversion and generation of ultra short pulses in passive mode locking schemes

Dr. Andrew Ellis was born in Underwood, England in 1965 and gained a BSc in Physics with a minor in mathematics from the University of Sussex, Brighton, England in 1987. He was awarded his $\mathrm{PhD}$ in Electronic and Electrical Engineering from The University of Aston in Birmingham, Birmingham, England in 1997 for his study on All Optical Networking Beyond $10 \mathrm{Gbit} / \mathrm{s}$.

He previously worked for British Telecom Research Laboratories as a Senior Research Engineer investigating the use of optical amplifiers and advanced modulation formats in optical networks and the Corning Research Centre as a Senior Research Fellow where he led activities in optical component characterisation. Currently, he heads the Transmission and Sensors Group at the Tyndall National Institute in Cork, Ireland, where he is also a member of the Department of Physics, University College Cork. He research interests include the evolution of core and metro networks, and the application of photonics to sensing.

Dr Ellis is a member of the Institute of Physics and the Institute of Engineering Technology, and is a Chartered Physicist. He acts as a reviewer for IEEE Journal of Lightwave Technology and Photonics Technology Letters and has published over 100 journal papers and over 20 patents in the field of Photonics.

Liam P. Barry received his BE (Electronic Engineering) and MEngSc (Optical Communications) degrees from University College Dublin in 1991 and 1993 respectively. From February 1993 until January 1996 he was employed as a Research Engineer in the Optical Systems Department of France Telecom's Research Laboratories (CNET) in Lannion, France, and as a result of this work he obtained his PhD Degree from the University of Rennes. In February 1996 he joined the Applied Optics Centre in Auckland University, New Zealand, as a Research Fellow. His work in New Zealand was mainly concerned with optical pulse generation and measurement, and the use of optical nonlinearities for high speed all-optical switching in fibre networks. In March 1998 he took up a lecturing position in the School of Electronic Engineering at Dublin City University, and established the Radio and Optical Communications Laboratory, which is part of the Research Institute for Networks and Communications Engineering (RINCE). He is currently Associate Professor in the School of Electronic Engineering, Director of RINCE, and a Principal Investigator for Science Foundation Ireland. His main research interests are; all-optical signal processing, optical pulse generation and characterization, hybrid radio/fibre communication systems, wavelength tuneable lasers for reconfigurable optical networks, and optical performance monitoring. 